\title{
DSM BUILDING SHAPE REFINEMENT FROM COMBINED REMOTE SENSING IMAGES BASED ON WNET-CGANS
}

\author{
Ksenia Bittner ${ }^{1}$, Marco Körner ${ }^{2}$, Peter Reinartz ${ }^{1}$ \\ ${ }^{1}$ Remote Sensing Technology Institute, German Aerospace Center (DLR), Wessling, Germany - \\ (ksenia.bittner, peter.reinartz)@ dlr.de \\ ${ }^{2}$ Technical University of Munich, Munich, Germany - marco.koerner@tum.de
}

\begin{abstract}
We describe the workflow of a digital surface models (DSMs) refinement algorithm using a hybrid conditional generative adversarial network (cGAN) where the generative part consists of two parallel networks merged at the last stage forming a WNET architecture. The inputs to the so-called WNETCGAN are stereo DSMs and panchromatic (PAN) half-meter resolution satellite images. Fusing these helps to propagate fine detailed information from a spectral image and complete the missing 3D knowledge from a stereo DSM about building shapes. Besides, it refines the building outlines and edges making them more rectangular and sharp.
\end{abstract}

Index Terms - Conditional generative adversarial networks, digital surface model, 3D scene refinement, 3D building shape, data fusion, satellite images

\section{INTRODUCTION}

A digital surface model (DSM) is an important and valuable data source for many remote sensing applications, like building detection and reconstruction, cartographic analysis, urban planning, environmental investigations and disaster assessment tasks. The use of DSM for those remote sensing applications is motivated by the fact that it already provides geometric descriptions about the topographic surface. With recent advances in sensor technologies, it became possible to generated DSMs with a ground sampling distance (GSD) smaller than $1 \mathrm{~m}$ not only from land surveying, aerial images, laser ranging data, or interferometric synthetic aperture radar $(\operatorname{InSAR})$, but also using satellite stereo images. The main advantages of satellite photogrammetric DSMs are the large land coverage and possibility to access remote areas. However, DSMs generated with the image-based matching approaches miss objects like steep walls in urban areas or feature some unwanted outliers and noise due to temporal changes, matching errors or occlusions. To overcome these problems, algorithms from computer vision have been analyzed and adapted to satellite imagery. For example, the filtering techniques such as geostatistical filter integrated with a hierarchical surface fitting technique, a threshold slope-based filter, or a Gaussian noise removal filter are the ones commonly used for DSM quality improvements. Moreover, some methodologies propose to fuse DSMs obtained from different data sources to compensate the limitations and gaps which each of them has individually [1].

With recent developments devoted to deep learning, it became possible to achieve top scores on many tasks including image processing. As a result, several works have already investigated their applicability for remote sensing applications, like landscape classification, building and road extraction, or traffic monitoring. Recently, a class of neural networks called generative adversarial networks (GANs) was applied on three-dimensional remote sensing data and proved to be suitable. Mainly, the generation of large-scale 3D surface models with refined building shape to the level of details (LoD) 2 from stereo satellite DSMs was studied using conditional generative adversarial networks (cGANs) [2,3]. In this paper, we follow those ideas and propose a hybrid cGAN architecture which couples half-meter resolution satellite panchromatic (PAN) images and DSMs to produce 3D surface models not only with refined 3D building shapes, but also with their completed structures, more accurate outlines, and sharper edges.

\section{METHODOLOGY}

The birth of GAN-based domain adaptation neural networks introduced by Goodfellow et al. [4] yielded great achievements in generating realistic images. The idea behind the adversarial manner of learning is to train a pair of networks in a competing way: a generator $G$ that tries to fool the discriminator to make the source domain look like the target domain as much as possible, and a discriminator $D$ that tries to differentiate between the target domain and the transformed source domain. Taking the source distribution as input instead of a uniform distribution and using this external information to restrict both the generator in its output and the discriminator in its expected input leads to the conditional type of GANs. The objective function for cGANs can be expressed through a two-player minimax game 


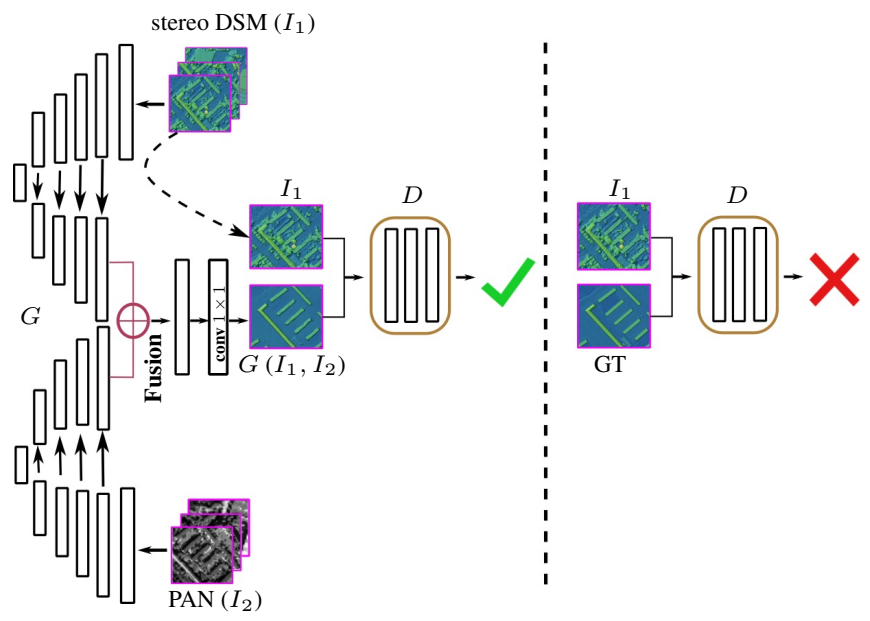

Fig. 1: Schematic overview of the proposed architecture for the building shape refinement in the $3 \mathrm{D}$ surface model by WNet-cGAN using depth and spectral information.

$$
G^{\star}=\arg \min _{G} \max _{D} \mathcal{L}_{\mathrm{cGAN}}(G, D)+\lambda \mathcal{L}_{L_{1}}(G)
$$

between the generator and the discriminator, where $G$ intents to minimize the objective function $\mathcal{L}_{\mathrm{cGAN}}(G, D)$ against the $D$ that aims to maximize it. Moreover, it should be mentioned that in the first term of Eq. (1) we use an objective function with least squares instead of the common negative log likelihood. The second term in Eq. (1) regularizes the generator and produces the output near the ground truth in a $L_{1}$ sense.

In our previous work, we already adapted the architecture proposed by Isola et al. [5] to obtain refined 3D surface models from the noisy and inaccurate stereo DSMs. Now, we propose a new cGAN architecture that integrates depth information from stereo DSMs together with spectral information from PAN images, as the latter provides a sharper information about building silhouettes, which allows not only a better reconstruction of building outlines but also their missing construction parts. Since intensity and depth information have different physical meanings, we propose a hybrid $G$ network where two separate UNet [6] type of networks with the same architecture are used: we feed one part with the PAN image and the second part with the stereo DSM generating a so-called WNet architecture. Before the last upsampling layer, which leads to the final output size, we concatenate the intermediate features from both streams. Moreover, we increase the network with an additional convolutional layer of size $1 \times 1$, which plays the role of information fusion from different modalities. As investigated earlier, this fusion can correct small failures in the predictions by automatically learning which stream of the network provides the best prediction result [7]. Finally, the tanh activation function $\sigma_{\tanh }(z)=\tanh (z)$ is applied on the top layer of the $G$ network. $D$ is represented by a binary classification network with a sigmoid activation function $\sigma_{\text {sigm }}(z)=\frac{1}{1+\mathrm{e}^{-z}}$ to the top layer to output the probability that the input image belongs either to class 1 ("real") or class 0 ("generated"). It has five convolutional layers which are followed by a leaky rectified linear units (ReLU) activation function

$$
\sigma_{\text {leaky ReLU }}(z)= \begin{cases}z, & \text { if } z>0 \\ a z, & \text { otherwise }\end{cases}
$$

with a negative slope $a$ of 0.2 . The input to $D$ is a concatenation of a stereo DSM with either a WNet-generated 3D surface model or a ground-truth DSM. A simplified representation of the proposed network architecture is demonstrated in Fig. 1.

\section{STUDY AREA AND EXPERIMENTS}

Experiments have been performed on WorldView-1 data showing the city of Berlin, Germany, within a total area of $410 \mathrm{~km}^{2}$. As input data, we used a stereo DSM and one of six very high-resolution PAN images, both with a resolution of $0.5 \mathrm{~m}$. The PAN image is orthorectified. As ground truth, the LoD2-DSM, generated with a resolution of $0.5 \mathrm{~m}$ from a city geography markup language (CityGML) data model, was used for learning the mapping function between the noisy DSM and the LoD2-DSM with better building shape quality. The detailed methodology on LoD2DSM creation is given in our previous work. A CityGML data model is freely available on the download portal Berlin 3D (http://www.businesslocationcenter.de/ downloadportal).

The implementation of the proposed WNet-cGAN is done with the PyTorch python package. For the training process, the satellite images were tiled into patches of size $256 \times 256$ px to fit into the single NVIDIA TITAN X (PASCAL) GPU with $12 \mathrm{~GB}$ of memory. The total number of epochs was set to 200 with a batch size of 5 . We trained the DSM-to-LoD2 WNet-cGAN network with minibatch stochastic gradient descent (SGD) using the ADAM optimizer. An initial learning rate was set to $\alpha=0.0002$ and the momentum parameters to $\beta_{1}=0.5$ and $\beta_{2}=0.999$.

\section{RESULTS AND DISCUSSION}

Two selected areas of the resulting LoD2-like DSM generated from combined spectral and depth information together with the LoD2-like DSM from a single image are illustrated in Fig. 2. From Fig. 2b and Fig. $2 \mathrm{~g}$ we can see that the refinement of building shapes only from stereo DSMs is a very challenging task, due to several reasons. First of all, the presence of vegetation can influence the reconstruction as some parts of buildings are covered by trees. Besides, the stereo DSM is very noisy itself, due to failures in the generation algorithms. It means that in most cases the types of roofs and, as a result, their shapes are indistinguishable. On the other hand, 


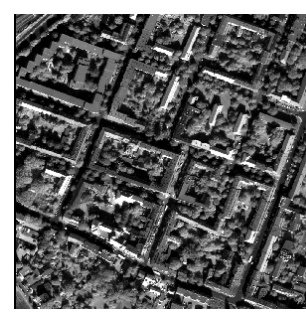

(a) PAN

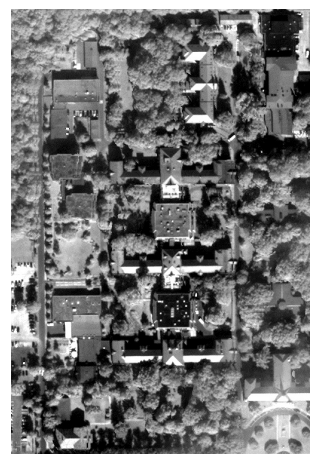

(f) PAN

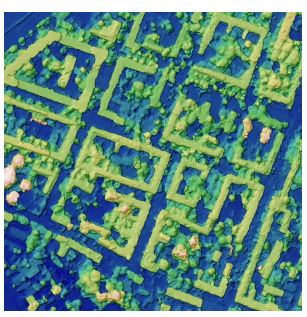

(b) Stereo DSM

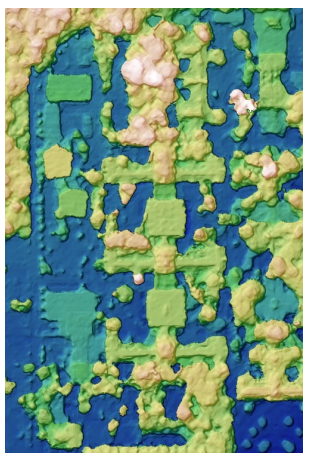

(g) Stereo DSM

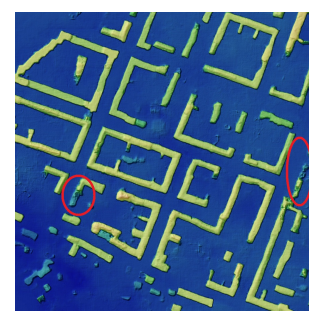

(c) cGAN LoD2-DSM

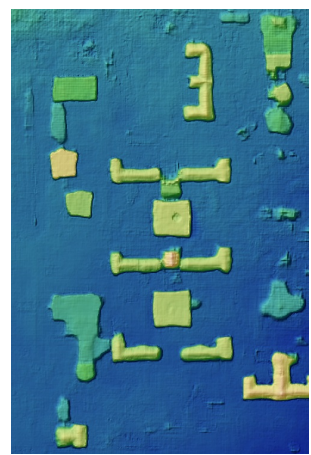

(h) cGAN LoD2-DSM

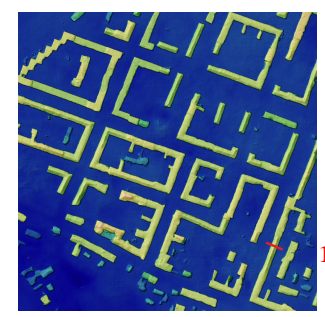

(d) WNet-cGAN LoD2-DSM

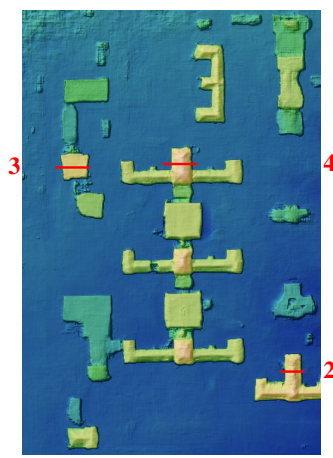

(i) WNet-cGAN LoD2-DSM

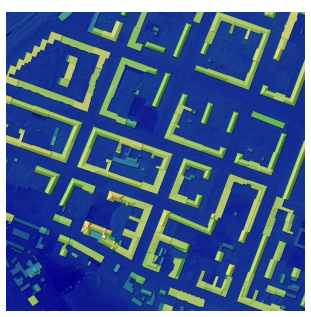

(e) GT: LoD2-DSM

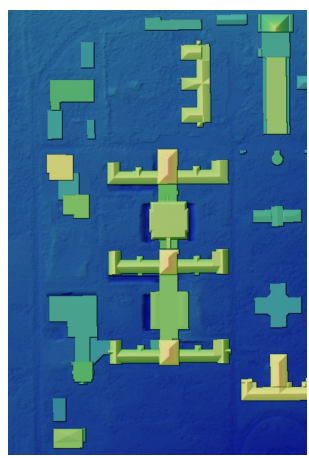

(j) GT: LoD2-DSM

Fig. 2: Visual analysis of DSMs, generated by stereo cGAN and WNet-cGAN architectures, over selected urban areas. The DSM images are color-shaded for better visualization.

looking at Fig. 2a and Fig. 2f we can see that the edges and outlines can be seen very well in the PAN image. Refinement of 3D buildings only from PAN image though would be very difficult as it does not contain 3D information, which is very important. Therefore, the combination of these two types of information is a good compromise which leads to advantages.

It can be clearly seen that the hybrid WNet-cGAN architecture is able to reconstruct more complete building structures than the cGAN from a single data source (see the highlighted buildings in Fig. 2d). Even complicated forms of buildings are also preserved in the reconstructed 3D surface model. The obvious example is a zigzag-shaped building at the upper-left part in Fig. 2d. This information could be only obtained from the PAN image (see Fig. 2a).

The second example depicts a smaller but scaled area for better visual investigation. Here, a central building is complete and more details are distinguishable. Besides, the ridge lines of the roofs are also much better visible. One can even guess to which type of roof parts of building belong to: gable or hip roofs. A clear contribution of the spectral information to the building shape refinement task can be seen at the upper-right building in Fig. 2i. We can notice that this building structure is more complete. The outlines of all buildings are clearer rectilinear and the building shapes become more symmetrical. To look more detailed into the 3D information, we illustrate some building profiles. We can see that the roof forms like gable and hip are clearly improved. The ridge lines tend to be sharp peaks. With the profile in Fig. 3d we again highlight the ability of the proposed architecture to reconstruct even complicated buildings, which is difficult to reconstruct using a single stereo DSM information.

To quantify the quality of the generated DSMs, we evaluated the metrics mean absolute error (MAE), root mean squared error (RMSE), normalized median absolute deviation (NMAD) and normalized correlation coefficient (NCC), commonly used for 3D surface model accuracy investigation, on the cGAN and WNet-cGAN setups and report their performance in Table 1. As we are interested in quantifying the improvements only of the building shapes on DSMs the above mentioned metrics were measured only within the area where buildings are present plus a three-pixel buffer around each of them. This was achieved by employing the binary building mask and dilation procedure on the footprint boundaries. From the obtained results we can see that DSM from WNet-cGAN is better than the original stereo DSM and the DSM generated by the cGAN model on all proposed metrics. This is reasonable, as the spectral information provides additional information, helpful to reconstruct building structure more accurately and detailed, which is not possible using only stereo DSM. This feature especially influences

Table 1: Prediction accuracies of cGAN and Fused-cGAN models on investigated metrics over the Berlin area.

\begin{tabular}{|c|c|c|c|c|}
\hline & MAE, $\mathbf{m}$ & RMSE, $\mathbf{m}$ & NMAD, $\mathbf{m}$ & NCC, $\mathbf{m}$ \\
\hline Stereo DSM & 3.00 & 5.97 & 1.48 & 0.90 \\
cGAN & 2.01 & 4.78 & 0.86 & 0.92 \\
Fused-cGAN & $\mathbf{1 . 7 9}$ & $\mathbf{4 . 3 6}$ & $\mathbf{0 . 6 7}$ & $\mathbf{0 . 9 4}$ \\
\hline
\end{tabular}




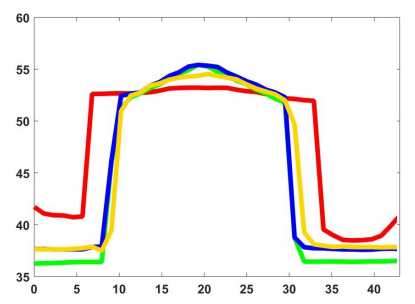

(a) Profile 1

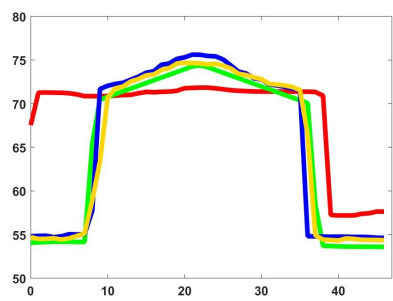

(b) Profile 3

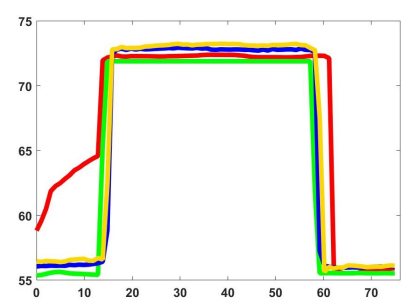

(c) Profile 4

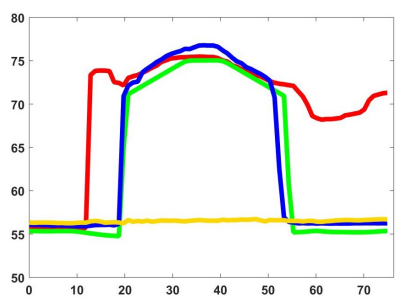

(d) Profile 5 Stereo DSM GT: LoD2-DSM cGAN LoD2-like DSM WNet-cGAN LoD2-like DSM

Fig. 3: Visual analysis of selected building profiles in generated DSMs.

the corners, outlines, and ridge lines. As the NCC metric indicates how the form of the object resembles the ground truth object, the gaining of $4 \%$ in comparison to the stereo DSM and $2 \%$ improvement on the DSM generated by cGAN model over the whole test area, which includes thousands of buildings, demonstrate the advantage of using complementary information for such complicated tasks. The high values of RMSE (order of $5 \mathrm{~m}$ ) is due to data acquisition time difference between the available DSM generated from stereo satellite images and the given ground truth data. As a result, several buildings are not presented or newly constructed in the more recent data set.

\section{CONCLUSION}

Refinement and filtering techniques from the literature for digital surface models (DSMs) quality improvement are adequate for either small-scale DSMs or DSMs with no discontinuities. As a result, there is a need to develop a refinement procedure that can handle discontinuities, mainly building forms in urban regions, in high-resolution large-scale DSMs. A common strategy in remote sensing for refinement procedures is the use of all available information from different data sources. Their combination helps to compensate the mistakes and gaps in each independent data source.

We present a method for automatic large-scale DSM generation with refined building shapes to the level of details (LoD) 2 from multiple spaceborne remote sensing data on the basis of conditional generative adversarial networks $(c G A N s)$. The designed end-to-end WNet-cGANs integrates the contextual information from height and spectral images to produce good-quality 3D surface models. The obtained results show the potential of the proposed methodology to generate more completed building structures in DSMs. The network is able to learn how to complement the strong and weak sides of panchromatic (PAN) image and stereo DSM, as, for instance, the stereo DSMs provide elevation information of the objects, but PAN images provide texture information and, as a result, more accurate building boundaries and silhouettes.

\section{References}

[1] M. Karkee, B. L. Steward, and S. A. Aziz, "Improving quality of public domain digital elevation models through data fusion," Biosystems Engineering, vol. 101, no. 3, pp. 293-305, 2008.

[2] K. Bittner, P dAngelo, M Körner, and P Reinartz, "Automatic large-scale $3 \mathrm{~d}$ building shape refinement using conditional generative adversarial networks," in Proceedings of the IEEE Conference on Computer Vision and Pattern Recognition (CVPR) Workshops, Salt Lake City, Utah, 2018, pp. 18-22.

[3] K. Bittner, P. dAngelo, M. Körner, and P. Reinartz, "Dsm-to-lod2: Spaceborne stereo digital surface model refinement," Remote Sensing, vol. 10, no. 12, p. 1926, 2018.

[4] I. Goodfellow, J. Pouget-Abadie, M. Mirza, B. Xu, D. Warde-Farley, S. Ozair, A. Courville, and Y. Bengio, "Generative adversarial nets," in Advances in neural information processing systems, 2014, pp. 2672-2680.

[5] P. Isola, J.-Y. Zhu, T. Zhou, and A. A. Efros, "Imageto-image translation with conditional adversarial networks," arXiv preprint arXiv:1611.07004, 2016.

[6] O. Ronneberger, P. Fischer, and T. Brox, "U-net: Convolutional networks for biomedical image segmentation," in International Conference on Medical Image Computing and Computer-Assisted Intervention, Springer, 2015, pp. 234-241.

[7] K. Bittner, F. Adam, S. Cui, M. Körner, and P. Reinartz, "Building footprint extraction from vhr remote sensing images combined with normalized dsms using fused fully convolutional networks," IEEE Journal of Selected Topics in Applied Earth Observations and Remote Sensing, vol. 11, no. 8, pp. 2615-2629, 2018. 